\title{
Fatigue strength characterization of Al-Si cast material incorporating statistical size effect
}

\author{
Roman Aigner ${ }^{1 *}$, Martin Leitner ${ }^{1}$, and Michael Stoschka ${ }^{1}$ \\ ${ }^{1}$ Christian Doppler Laboratory for Manufacturing Process based Component Design, Montanuniversität Leoben, Chair of Mechanical \\ Engineering, 8700 Leoben, Austria
}

\begin{abstract}
Cast aluminium components may exhibit material imperfections such as shrinkage and gas pores, or oxide inclusions. Therefore, the fatigue resistance is significantly influenced by the size and location of these inhomogenities. In this work, two different specimen geometries are manufactured from varying positions of an $\mathrm{Al}-\mathrm{Si}-\mathrm{Cu}$ alloy casting. The specimen geometries are designed by means of shape optimization based on a finite element analysis and exhibit different highly-stressed volumes. The numerically optimized specimen curvature enforces a notch factor of only two percent. To enable the evaluation of a statistical size effect, the length of the constant testing region and hence, the size of the highly-stressed volume varies by a ratio of one to ten between the two specimen geometries. Furthermore, the location of the crack initiation is dominated by the comparably greatest defects in this highly-stressed volume, which is also known as Weibull's weakest link model. The crack initiating defect sizes are evaluated by means of light microscopy and modern scanning electron microscope methods. Finally, the statistical size effect is analysed based on the extreme value distribution of the occurring defects, whereby the size and location of the pores is non-destructively obtained by computed tomography (CT) scanning. This elaborated procedure facilitates a size-effect based methodology to study the defect distribution and the associated local fatigue life of CPS casted Al-Si lightweight components.
\end{abstract}

\section{Introduction}

Aluminium cast parts enable quite complex geometries thus featuring lightweight goals. On the other hand, the service strength must be well known already in early stages of the design process. For such applications, Al$\mathrm{Si}-\mathrm{Cu}$ cast alloys are commonly used materials. One of the most important limiting factors regarding fatigue strength is the size of the intrinsic defects in the casting. Microstructural parameters like the grain size or the dendrite arm spacing (DAS) show a secondary influence [1]. Numerous preliminary studies showed an influence of the solidification time of the melted aluminium on the dendrite arm spacing, and hence, additionally on the resulting fatigue strength of the bulk material [2]. The dominant material flaws, who scale as well with the local solidification time, cause this.

As exemplified in other preliminary studies [3, 4], Tiryakioglu showed that in the HCF region a statistic model can be applied to link the fatigue life with the area of the fracture-initiating micropores [5]. The aim of this work is to investigate the fatigue strength of $\mathrm{Al}-\mathrm{Si}-\mathrm{Cu}$ cast alloy at three sampling positions, whereby each series exhibits two varying highly-stressed volumes. In addition, $\mu \mathrm{CT}$ scans of the differently sized specimens are compared by means of the location and size of the micropores. Furthermore, the fracture surfaces of the tested specimens are subsequently analysed to gain information about the crack initiating pores, their position as well as their spatial extent. Subsequently, the extreme value distribution of the fracture-initiating defects are evaluated on the basis of a most likelihood type of function.

\section{Experimental}

\subsection{Investigated Material}

The examined material is an $\mathrm{Al}-\mathrm{Si}-\mathrm{Cu}$ cast alloy in $\mathrm{T} 6$ heat-treated condition. The specimens are manufactured from six different locations, which possess by simulation only minor differences in cooling rate. They are arranged into two regions featuring specimens of different sizes for examination of size effect. The two regions exhibit a similar local cooling rate and therefore, a similar DAS is expected. Measurements on metallographic sections revealed that the DAS in these two testing regions is nearly identical; see fig. [1].

This supports the assumption that the subsequent experimental and analytical investigations focus on the size effect and are not affected by local microstructural parameters.

* Corresponding author: roman.aigner@unileoben.ac.at 


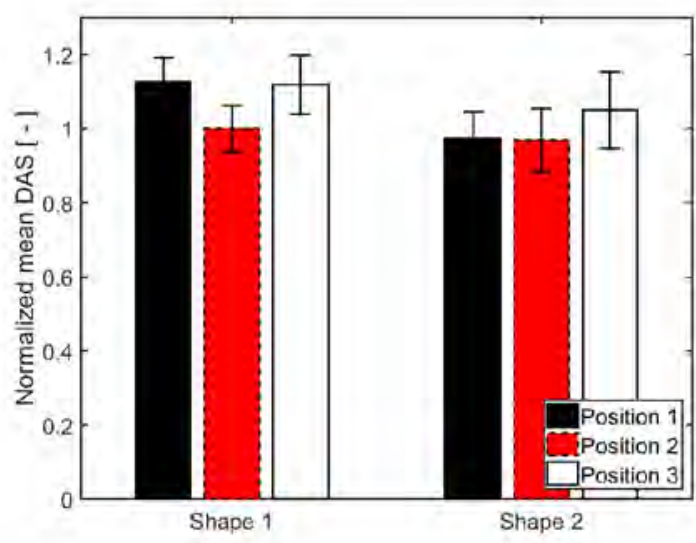

Fig. 1. Microstructural properties in the testing region

As aforementioned, the two different specimen shapes vary only by means of the highly-stressed volume. The larger specimens are labelled as shape 2. The constant testing diameter ensures a specimen failure at the most critical defect, thus depending on its size, spatial location and orientation, in agreement with Weibull's WeakestLink concept. As seen in fig. [2], due to variation of the testing length between the two specimen shapes, the ratio of the highly-stressed volumes exhibits a value of about ten if evaluating stresses down to ninety-five percent of the peak values. Therefore, the statistical size effect for this cast aluminium alloy is properly determinable with these two specimen designs.

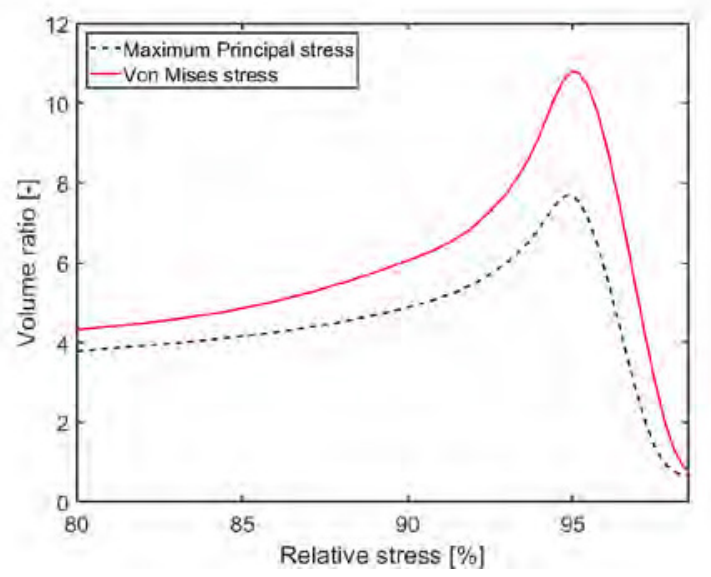

Fig. 2. Volume ratio of the varying specimen shapes (shape 1 to shape 2)

To assess the fatigue strength of the investigated material, quasi-static tensile tests are performed. These tensile test specimens are taken out of shape 2 position thus ensuring identical microstructural parameters.

Table 1. Normalized mechanical and microstructural properties of the investigated positions.

\begin{tabular}{|c|c|c|c|c|}
\hline Position & UTS [ - ] & YS [ - ] & $\mathbf{A}_{\mathbf{5 6 5}}[\mathbf{\%}]$ & DAS [ - ] \\
\hline 1 & 3.35 & 2.84 & 1.62 & 0.97 \\
\hline
\end{tabular}

\begin{tabular}{|l|l|l|l|l|}
\hline 2 & 3.30 & 2.78 & 1.62 & 0.96 \\
\hline 3 & 3.20 & 2.80 & 1.08 & 1.05 \\
\hline
\end{tabular}

The results, listed in tbl. [1], show that all three positions inherit similar quasi-static characteristics by means of the ultimate tensile strength and yield strength. On the other hand, the ductility of position 3 , represented by the elongation after fracture, is lower than on the other two positions, due to the higher porosity in position 3 . But yield strength and tensile strength are almost identical, only the DAS value at this position is slightly increased, though it differs only in the scale of standard deviation. Summing up, the evaluated DAS scatter index is about ten percent, but possible effects in extremal pore distribution are further on discussed.

\subsection{Fatigue results}

High-Cycle Fatigue tests are performed with each of the two specimen shape geometries. The tests are executed at a load ratio of $\mathrm{R}=-1$ under compression-tension loading with a testing frequency of about $100 \mathrm{~Hz}$. The run-out cycle number is set at ten million load-cycles because preliminary studies confirmed that defect-based specimen failures occur also in the endurance region between one and five million cycles. The slope of the high cycle fatigue region $\mathrm{k}_{2}$ of the $\mathrm{S} / \mathrm{N}$ curve is assumed to be five times as high as the slope $\mathrm{k}_{1}$ in the high cycle fatigue region as proposed in [6]. The evaluated fatigue data is statistically assessed in the finite life area invoking ASTM E739[7]. In addition, the fatigue strength in the transition region is evaluated by means of the $\arcsin \sqrt{p}$ methodology [8].

The results of the fatigue tests regarding specimen shape 1 show, that the fatigue strength differs only slightly between the three sampling positions, as seen in fig. [3].

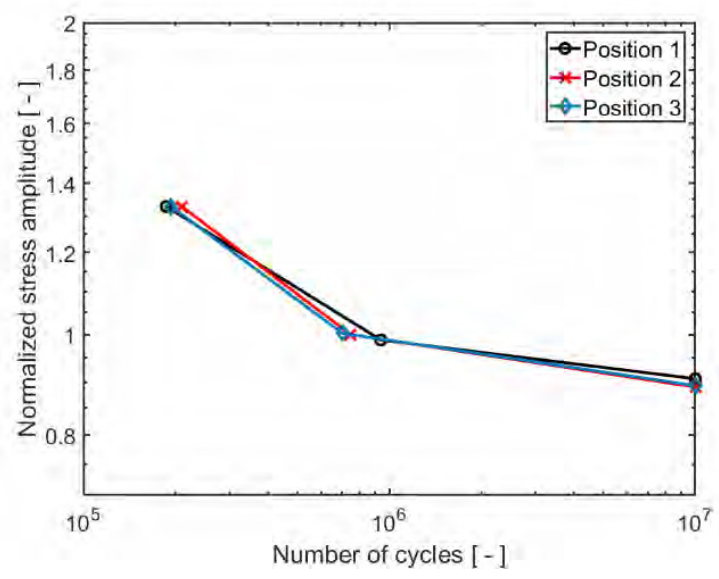

Fig. 3. Fatigue data from specimen shape 1 at different positions

As depicted in fig. [4], the specimens with shape 2 exhibit a distinctive lower fatigue strength than those with shape 1 . This reduction is expected as the highly- 
stressed volume is ten times larger in case of specimen with shape 2 compared to shape 1 .

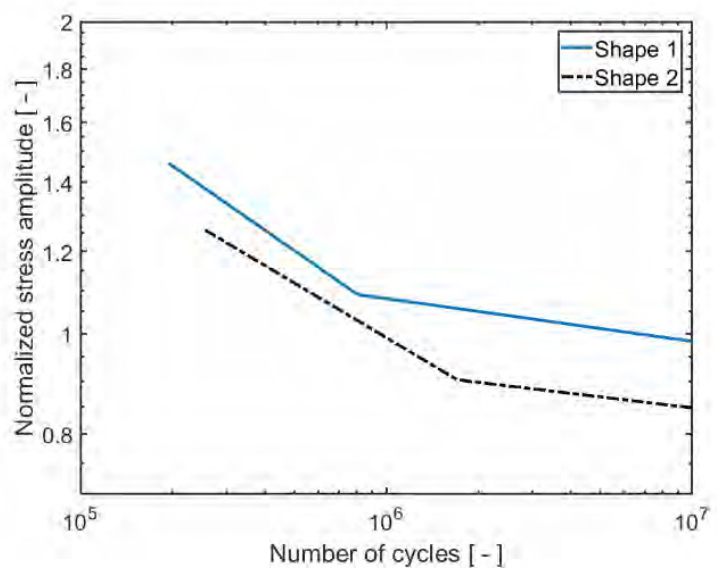

Fig. 4. Fatigue data commonly evaluated from all positions for two different specimen shapes to study size effect

This goes along with the statistical size effect, implying the elevated probability of shape 2 specimens to include greater defects than in shape 1, as shown in [9]. It must be pointed out that the results shown in fig. [4] incorporate all specimens of the three different positions, separated only by their geometric shape. The assessment of the shape 2 specimen $\mathrm{S} / \mathrm{N}$ curves shows a slightly higher scatter of the fatigue life compared to shape 1 . Furthermore, the slope and transition knee point $\mathrm{N}_{\mathrm{K}}$ of the shape 2 Wöhler curve is somewhat increased. In tbl. [2], the fatigue data of the six test series is listed in detail.

Table 2. Fatigue data from the varying sample positions.

\begin{tabular}{|c|c|c|c|c|}
\hline Position & Shape & $\mathbf{k}_{\mathbf{1}}$ & $\mathbf{N}_{\mathbf{K}}$ & $\mathbf{S}_{\mathbf{a}, \mathbf{P 5 0}, \mathbf{L L F}}$ \\
\hline 1 & 1 & 5.45 & $0.938 \mathrm{E} 6$ & 0.99 \\
\hline 2 & 1 & 4.46 & $0.747 \mathrm{E} 6$ & 1.00 \\
\hline 3 & 1 & 4.57 & $1.084 \mathrm{E} 6$ & 0.98 \\
\hline 1 & 2 & 6.65 & $1.993 \mathrm{E} 6$ & 0.87 \\
\hline 2 & 2 & 5.54 & $1.615 \mathrm{E} 6$ & 0.86 \\
\hline 3 & 2 & 5.07 & $1.210 \mathrm{E} 6$ & 0.83 \\
\hline
\end{tabular}

As exemplified in [2], the microstructure, by means of the DAS, has a significant influence on spatial extent of the immanent defects.

In case of increased dendrite arm spacings, an augmented sealed-off volume between two adjacent solidified dendrite arms is obtained thus increasing the likelihood of shrinkage pores. Numerous metallographic analyses are performed, which confirm the statistical relationship between the DAS and microporosity. In this context, the pore size is denoted as estimated mean equivalent circle diameter (ECD). The ECD is evaluated by the projected area of the defect in fractographic analysis and can be mathematically described by Eq (1).

$$
\mathrm{ECD}=\sqrt{\frac{4 \text { Area }}{\pi}}
$$

\section{Evaluation of defects}

\subsection{Fractography}

In order to locate the fracture-initiating defect and to evaluate its geometrical characteristics, each tested specimen is subsequently analysed by either digital microscope or scanning-electron-microscopy (SEM). The geometrical shape of each defect is manually determined by applying commercial image analysis software and further on statistically evaluated.

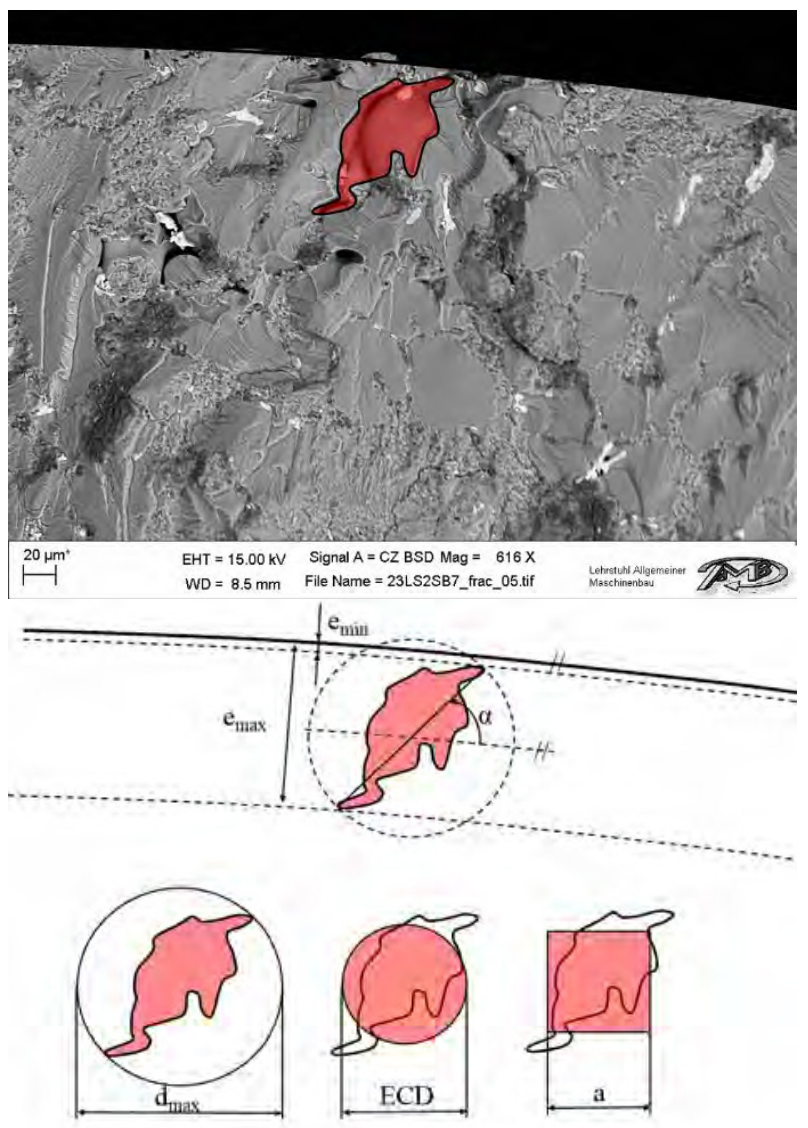

Fig. 5. Representative crack-initiating micropore and its geometrical characterization

Fig. [5] shows the crack initiation at a representative micropore. In addition, geometrical parameters such as the square root of the effective defect area a, its largest extent $\mathrm{d}_{\max }$, the equivalent circle diameter (ECD) and the minimal respectively maximum rectangular distance to the surface $\left(\mathrm{e}_{\min }, \mathrm{e}_{\max }\right)$ are evaluated accordingly. The orientation of the flaws is determined by means of the angle $\alpha$, which is measured between the largest 
geometric extent of the project defect area and the specimen surface. The analysis of the initiating cracks of shape 1 specimen deduces that in most cases the technical crack initiates at surface near defects. In detail, fig. [6] displays that the crack initiating defects are mostly surface-intercepting as the normalized minimum distance gets close to zero. This implies that these pores are truncated during the final machining process of the round specimens.

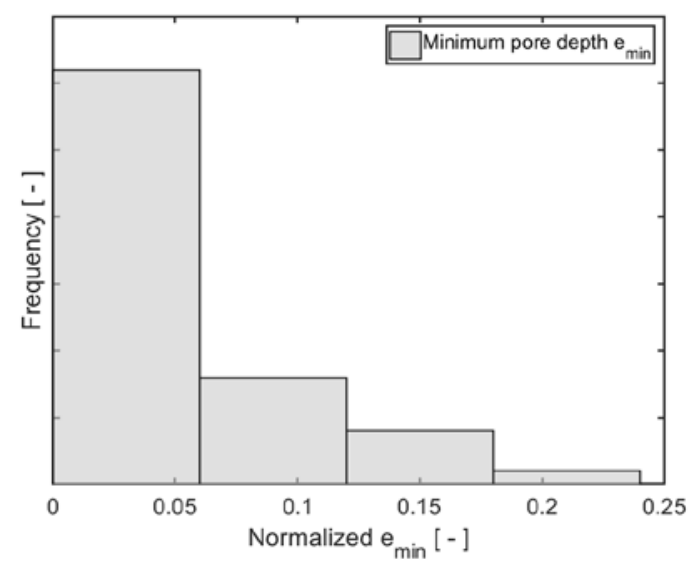

Fig. 6. Frequency histogram of the minimum pore depth $\mathrm{e}_{\min }$

Subsequently, these intrinsic defects can be treated like predominant surface cracks, whereat the crack initiation phase is lowered compared to closed defects within the volume. A study to evaluate the lifetime within the crack-initiating phase on microstructural defects is scheduled as further work.

In case of higher loaded specimens, load level at about twenty percent enhanced of the normalized stress amplitude, a second failure mechanism is recognizable. The crack starts from a surface-near microdefect until it progresses through large slip plane areas.

To characterize the local fatigue strength of near surface cracks, the determined geometric values can be used as initial approach. As the micropores are often cut-open, the maximum spatial extent $\mathrm{e}_{\max }$ of the defect can be considered as the intrinsic crack length. Fig. [7] shows the frequency distribution of the maximum spatial extent $\mathrm{e}_{\max }$. It also illustrates a fit of the probability density function of type one generalized extreme value distribution. This type one distribution, also known as Gumbel distribution [10, 11], possesses the following probability density function (pdf), see Eq (2).

$$
f(x)=\frac{1}{\sigma} \exp \left(-\frac{x-\mu}{\sigma}\right) \exp \left[-\exp \left(-\frac{x-\mu}{\sigma}\right)\right]
$$

Herein, $x$ represents the pore size, by means of $e_{\max }, \mu$ is the location parameter and $\sigma$ is the scale parameter of the distribution. Tiryakioglu showed [5] that the relationship between fatigue life and the corresponding defect size in Al-Si cast alloys can be well described by means of a Gumbel distribution. Furthermore, the area and hence the ECD of the fracture initiating micropores is evaluated. Therefore, the scale and the location parameters of the Gumbel distribution can be investigated by means of several different methodologies, including the method of moments, the maximum likelihood method and the least square method. According to [12], the maximum likelihood method is suited to be the best matching method to meet both parameters $\mu$ and $\sigma$.

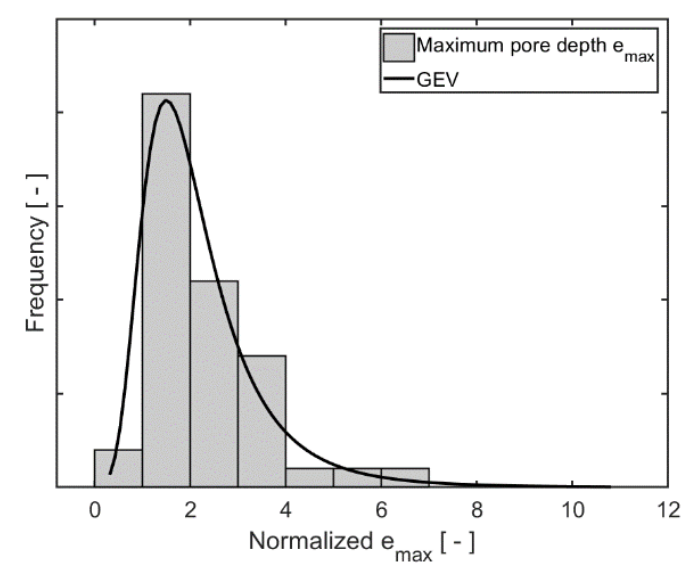

Fig. 7. Frequency histogram of the maximum pore depth $\mathrm{e}_{\max }$

In table tbl. [3], the different normalized parameters as well as their relating ninety-five percent confidence intervals for both shape 1 and shape 2 specimens are given. All the distribution parameters are evaluated on the basis of the maximum likelihood method, as recommended in [13].

Table 3. Estimated distribution parameters incorporating the $95 \%$ confidence intervals.

\begin{tabular}{|c|c|c|c|c|c|c|}
\hline \multirow{2}{*}{ Shape } & \multirow{\mu}{*}[-]{} & $\boldsymbol{\sigma}$ & \multicolumn{2}{|c|}{$\mathbf{C I}(\boldsymbol{\mu})[-]$} & \multicolumn{2}{|c|}{$\mathbf{C I}(\boldsymbol{\sigma})[-]$} \\
\cline { 4 - 7 } & {$[-]$} & $\mathbf{5 \%}$ & $\mathbf{9 5} \%$ & $\mathbf{5 \%}$ & $\mathbf{9 5 \%}$ \\
\hline 1 & 2.09 & 0.30 & 1.18 & 1.44 & 0.22 & 0.41 \\
\hline 2 & 1.31 & 0.33 & 1.69 & 2.47 & 0.13 & 0.84 \\
\hline
\end{tabular}

The cumulative distribution function (cdf) for the Gumbel distribution of both specimen shapes is subsequently calculated as denoted in Eq. (3). It is displayed along with the probability density function in fig. [8]. The maximum occurring defect size elevates the density function, which is in agreement to Weibull's Weakest-Link concept [14] as specimens of shape 2 possess an increased highly-stressed volume.

$$
F(x)=\exp \left[-\exp \left(-\frac{x-\mu}{\sigma}\right)\right]
$$

The probability density function of both tested sample volumes also show a similar shape parameter $\sigma$. In detail, specimen geometries of shape 1 lead to a value of $\sigma=0.30$ and a value of 0.32 for shape 2 samples, which 
confirms the assumption that the cast manufacturing process is equal for both shape geometries.

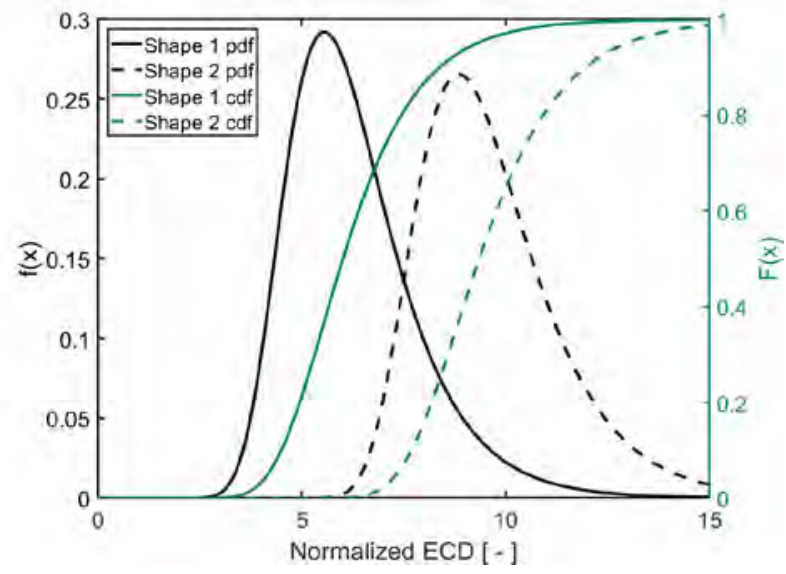

Fig. 8. Probability distributions of both tested specimen shapes possessing two different highly-stressed volumes

\subsection{Computed tomography}

In order to characterize not only the fracture initiating defect but also the completely tested volumina in terms of intrinsic inhomogenities, non-destructive high resolution $\mu \mathrm{CT}$ scans at selected samples are performed. Due to the low density of the tested material, the transmission by means of radiography is comparably high. Therefore, defects of about two to three times the voxel size can be properly evaluated. One voxel in CTbased image analysis refers to one pixel in the threedimensional space. This defines the resolution of the $\mu \mathrm{CT}$ scan. Fig. [9] demonstrates an X-ray tomography scan result of a representative testing volume.

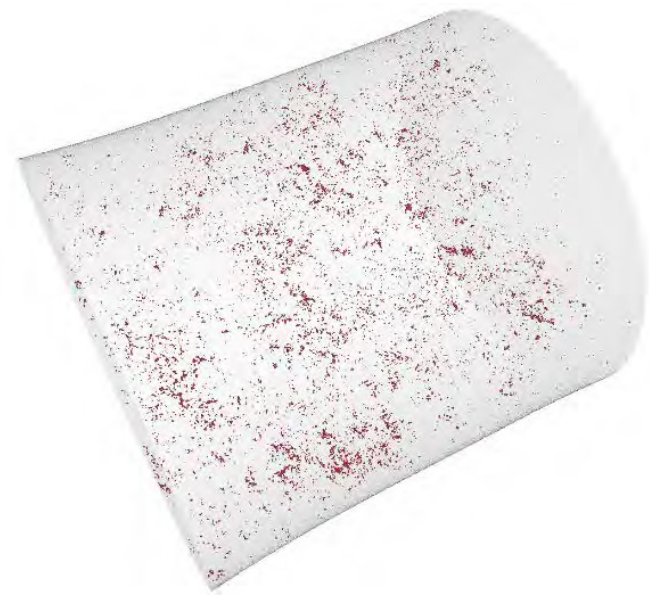

Fig. 9. $\mu \mathrm{CT}$ scan of a representative specimen

In numerous preceding studies e.g. $[15,16]$, the standards for computed tomography investigations as well as the description of experimental procedures and the subsequent image analysis are investigated. It is important to scan the tested volume with a reasonably high resolution to evaluate the micropore shape accordingly.
Thus a voxel size of about $5.5 \mu \mathrm{m}$ is applicable. The assessed spatial extent of the defects by $\mu \mathrm{CT}$ reveals that the evaluated size of the micropores is somewhat larger than by means of fractographic analysis. This results from the location of the defects. As previously mentioned, the crack-initiating pores are mostly in the surface near region though not necessarily being the largest defect in the tested volume.

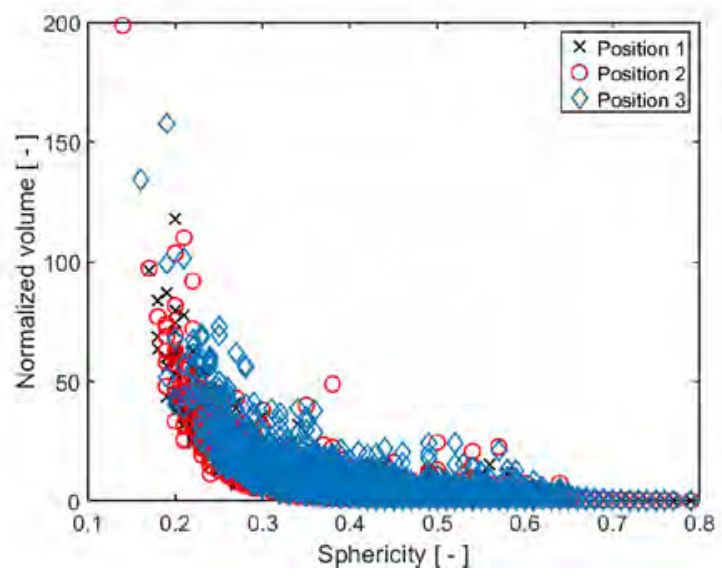

Fig. 10. Sphericity of the flaws in specimen shape 1

On the one hand, the fatigue limit of the bulk material slightly decreases in the surface layers due to a lower dislocation density. Furthermore, flaws with an orientation $\alpha$ close to ninety degrees inherit a faster crack propagation due to its dominant growth direction towards the interior of the specimen. Subsequently, the cut-open pores act like a predominant crack and inherit a shorter crack-initiating phase.

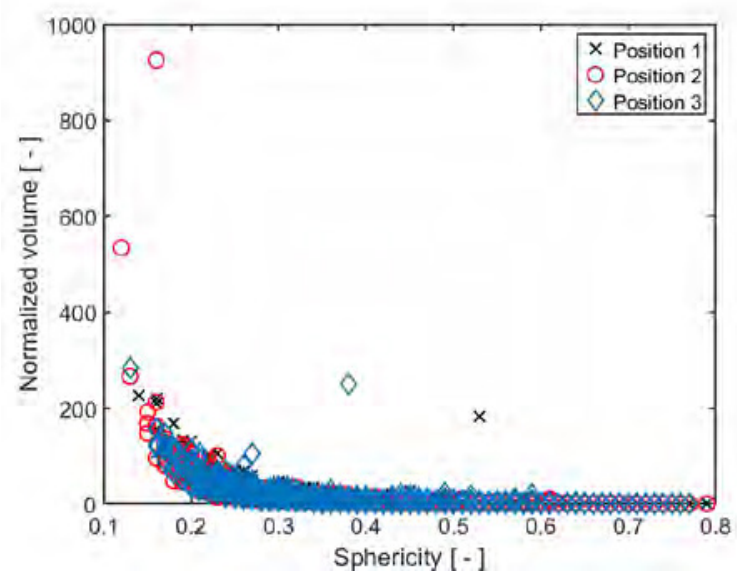

Fig. 11. Sphericity of the flaws in specimen shape 2

Finally, the size effect on pore sphericity can be evaluated. The investigated volumes show that the sphericity of all $\mu \mathrm{CT}$-detected micropores decreases with lowered volume. As one can see in fig. [10] and fig. [11], larger defects possess a rather sharp needleshaped structure, while the smaller defects are more spherical.

These figures additionally highlight that differences in extremal imperfection size exist for the two evaluated 
specimen shapes. In detail, the extreme volume is about four times if the highly-stressed volume ratio is set ten to one.

\subsection{Statistical size effect}

To study the statistical size effect, the ratio between the highly-stressed volume $\mathrm{V}_{2}$ of shape 2 is ten times the highly-stressed volume $\mathrm{V}_{1}$ of shape 1 . Therefore, the probability to contain larger defects of $\mathrm{V}_{2}$ increases [1719]. The relationship between the volume size and the maximum intrinsic defect size is given by the Gumbel distribution; see Eq. (2). The cumulate probability distribution function $\mathrm{F}(\mathrm{x})$ defines the probability of occurrence for a certain imperfection size $\mathrm{P}(\mathrm{x})$ within the examined volume. The ratio between the highly-stressed volume and the reference control volume is denoted as return period $T$. The probability that the variable $x$ exceeds the return variable $\mathrm{X}_{\mathrm{t}}$ is given as $\mathrm{P}(\mathrm{x})$. If the variables $X_{1}, X_{i} \ldots X_{n}$ are a sequence of random variables representing the maximal size of a defect within a given volume $\mathrm{V}$, then the maximal size $\mathrm{Y}$ in an increased volume $V_{n}$ is given by Eq. (4).

$$
Y=\max \left\{X_{1}, X_{i}, \ldots X_{n}\right\}
$$

The probability of a n- times greater volume is given by Eq. (5), postulating a Gumbel type extreme value distribution leads to Eq. (6). Therefore, the fatigue life of a corresponding specimen with a larger tested volume is proportionally reduced.

$$
\begin{aligned}
& P_{n}(x)=P(x)^{n} \\
& P_{n}(x)=\exp \left[-\exp \left(-\frac{x-\mu}{\sigma}\right)\right]^{n}
\end{aligned}
$$

Another method to describe the statistical size effect, as suggested in $[20,21]$, is based only on the highlystressed volume, as it features an elevated probability of crack initiations. The two presented models differ only in the relative stress limit of the highly-stressed volume; in detail Sonsino suggests to use the ninety percent limit whereas Kuguel recommends to take the volume with relative stresses above ninety-five percent into account. Both models are mathematically described by the following Eq. (6), in which $\mathrm{V}_{\mathrm{n}}$ represents the limit value of the highly-stressed volume.

$$
\frac{\sigma_{\mathrm{a}, \mathrm{V} 1}}{\sigma_{\mathrm{a}, \mathrm{V} 2}}=\left(\frac{\mathrm{V}_{\mathrm{n}, 1}}{\mathrm{~V}_{\mathrm{n}, 2}}\right)^{\kappa}
$$

The material dependent parameter $\kappa$, acts as Weibull parameter and can be calculated from Eq. (8), depending on the probability distribution of the fatigue data [22]. According to Eq. (8), the calculated Weibull parameter $\kappa$ has a value of about 11.2, based on the evaluated fatigue scatter index $\mathrm{T}_{\mathrm{S}}$. It should be noted that other guidelines [23] defines the parameter as a constant $\kappa=10$ for aluminium castings.

$$
\kappa=\frac{1.3151}{\log \left(T_{S}\right)}
$$

A more thorough comparison of the different models to describe a statistical size effect in the high cycle fatigue region is given in [24].

\section{Results and analysis}

As listed in table tbl. 3, the investigated fatigue strength shows a significant distinction between both specimen shapes. In order to assess the statistical size effect, the fatigue strength is evaluated at a survival probability of ninety percent instead of using the proposed mean value. The evaluation takes all sample positions commonly into account as microstructural differences are negligible. The influence of the highly-stressed volume as statistical size effect is calculated by means of Eq. (7). Furthermore, the Weibull exponent is conservatively defined as $\kappa=10$, as recommended.

Fig. [12] demonstrates the adapted model, based on the volumetric approach from Sonsino [18]. The model calculates the expected fatigue strength levels for the two investigated specimen shapes quite exactly. In detail, the deviation for specimen shape 2 is only about $0.7 \%$ lower compared to the experiments. Therefore, the approach according to Sonsino in combination with an increased survival probability can be further used to assess the statistical size effect in Al-Si-Cu cast alloys.

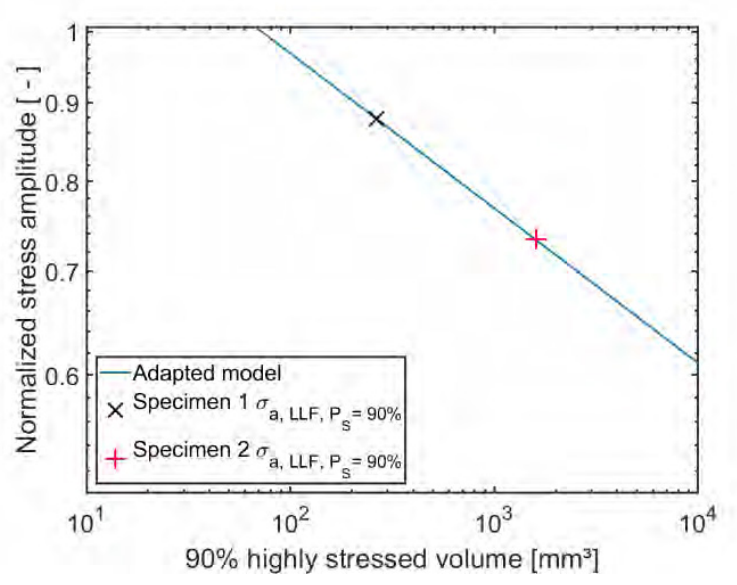

Fig. 12. Calculated fatigue strength in dependence of highly-stressed volume

\section{Conclusions}

This paper assesses the defect-correlated fatigue strength of Al-Si-Cu cast alloy incorporating the effect of the sampling position as well as the highly-stressed volume as statistical size effect. The fatigue tests are performed under alternating tension-compression. The different sampling positions and specimen shapes inherit similar microstructural properties to ensure only minor influences of varying DAS as microstructural parameter. 
Thus, the technological size effect is minimized but the presented test data can be used as reference in future work.

The fatigue test data reveals that the fatigue limit decreases by increasing the highly-stressed volume. Subsequent fractographical analysis is comparative conducted to characterize the crack initiating defects. Moreover, $\mu \mathrm{CT}$ scans are performed to investigate the defect population within the tested volumina, whereat the shape and spatial extent of the pores are assessed. Based on the evaluated defect data, the different probability distributions of both specimen shapes are derived by maximum likelihood regression. The obtained location and scale parameters of the extreme value distribution function are nearly identical for both specimen shapes, which confirms the assumption that the technological effect is negligible and the subsequent evaluation focusses only on the statistical size effect. Finally, a fatigue life model to assess the statistical size effect based on Weibull's approach is adopted towards higher probability of survival, which matches quite well and reveals only a minor difference with an error of about $0.7 \%$ compared to the experiments.

The financial support by the Austrian Federal Ministry for Digital and Economic Affairs and the National Foundation for Research, Technology and Development is gratefully acknowledged. Furthermore, I would like to thank the industrial partners BMW München Group and Nemak Dillingen $\mathrm{GmbH}$ for the excellent mutual scientific cooperation within the CD-laboratory framework.

\section{References}

1. A.M.A. Mohamed, F.H.Samuel, Saleh Al kahtani, Mat. Sci. \& Eng. A 577, (2013)

2. R. Minichmayr, W. Eichlseder, Giessereirundschau, 51, (2004)

3. C. Garb, M. Leitner, F. Grün, Procedia Eng., 160, (2016)

4. M. Leitner, C. Garb, H. Remens, M. Stoschka, Mat. Sci. \& Eng., A 707, (2017)

5. M. Tiryakioglu, The Minerals, Met. And Mat. Society and ASM International, 40, (2009)

6. H. Leitner, PhD Thesis, (2001)

7. ASTM International ASTM E739, (1998)

8. D. Dengel, Journal of Materials Technology, 8, (1975)

9. H. Friederich, B. Kaiser, K. H. Kloos, Mat.-wiss. U. Werkstofftech., 29, (1998)

10. E.J. Gumbel, Statistics of Extremes (1958)

11. B.V. Gnedenko, Ann. Math., 44, (1943)

12. S. Beretta, Y. Murakami, Fatigue \& Fract. of Mat. \& Struct., 21, (1998)

13. S. Mahdi, M. Cenac, Revista de Matematica, 12, (2005)

14. W. Weibull, Ingenieur Archiv, 2, (1959)
15. DIN EN 16016, (2011)

16. VDI/VDE-Richtlinie 2630, (2009)

17. K.D. Tocher, E.J. Gumbel, Journal of the Royal Statistical Society, (1955)

18. K. Heckel, J. Köhler, Materialwissenschaft und Werkstofftechnik, 6, (1975)

19. M. Tiryakioglu, Mat. Sci. and Eng. A 497, (2008)

20. C.M. Sonsino, Materialwissenschaft und Werkstofftechnik, 36, (2005)

21. R. Kuguel, ASTM Proceedings, 61, (1961)

22. J. W. Bergmann, A. Diemar, K. Linne, M. Vormwald, FVV, 784, (2004)

23. FKM Richtlinie, 6, (2012)

24. T. Tomaszewski, J. Sempruch, T. Piatowski, Journal of applied mechanics, 52, (2014) 